\title{
MOMENT-ANGLE COMPLEXES AND COMBINATORICS OF SIMPLICIAL MANIFOLDS
}

\author{
VICTOR M. BUCHSTABER AND TARAS E. PANOV
}

Let $\rho:\left(D^{2}\right)^{m} \rightarrow I^{m}$ be the orbit map for the diagonal action of torus $T^{m}$ on the unit poly-disk $\left(D^{2}\right)^{m} \subset \mathbb{C}^{m}$. Each face of the cube $I^{m}=[0,1]^{m}$ (viewed as a cubical complex) has the form

$$
F_{I \subset J}=\left\{\left(y_{1}, \ldots, y_{m}\right) \in I^{m}: y_{i}=0 \text { if } i \in I, y_{j}=1 \text { if } j \notin J\right\},
$$

where $I \subset J$ are two subsets of the index set $[m]=\{1, \ldots, m\}$. For each face $F_{I \subset J}$ put $B_{I \subset J}:=\rho^{-1}\left(F_{I \subset J}\right)$. If $\# I=i, \# J=j$, then $B_{I \subset J} \cong\left(D^{2}\right)^{j-i} \times T^{m-j}$.

Definition 1. Let $C$ be a cubical subcomplex of $I^{m}$. The moment-angle complex ma $(C)$ is the $T^{m}$-invariant decomposition of the subset $\rho^{-1}(|C|) \subset\left(D^{2}\right)^{m}$ into blocks $B_{I \subset J}$ corresponding to the faces $F_{I \subset J}$ of complex $C$.

Many combinatorial problems concerning cubical complexes may be treated by studying the equivariant topology of moment-angle complexes. In the present paper we realize this approach in the case of cubical complexes determined by simplicial complexes. Let $K^{n-1}$ be an $(n-1)$-dimensional simplicial complex with $m$ vertices, and $|K|$ the corresponding polyhedron. If $I=\left\{i_{1}, \ldots, i_{k}\right\} \subset[m]$ is a simplex of $K$, then we would write $I \in K$. Define the following two cubical subcomplexes of $I^{m}$ :

$$
\operatorname{cub}(K)=\left\{F_{I \subset J}: J \in K, I \neq \varnothing\right\}, \quad \operatorname{cc}(K)=\left\{F_{I \subset J}: J \in K\right\} .
$$

Lemma 2. As a topological space, the complex $\operatorname{cub}(K)$ is homeomorphic to $|K|$, while $\operatorname{cc}(K)$ is homeomorphic to the cone $|\operatorname{cone}(K)|$.

The cubical complex $\operatorname{cc}(K)$ was introduced in [1] and then studied in [2]. The cubical complex $\operatorname{cub}(K)$ appeared in [3].

a)

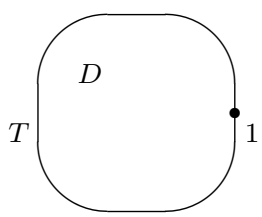

b)

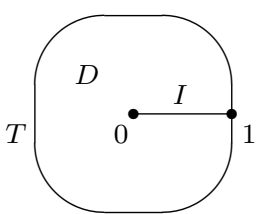

Denote the moment-angle complexes corresponding to $\operatorname{cub}(K)$ and $\operatorname{cc}(K)$ by $\mathcal{W}_{K}$ and $\mathcal{Z}_{K}$ respectively. Consider the cellular decomposition of the poly-disk $\left(D^{2}\right)^{m}$ that is obtained by subdividing each factor $D^{2}$ into 0 -dimensional cell 1, 1-dimensional cell $T$, and 2-dimensional cell $D$, see Fig. a). Each cell of $\left(D^{2}\right)^{m}$ is a product of cells $D_{i}, T_{i}, 1_{i}$, $i=1, \ldots, m$, i.e., can be written as $D_{I} T_{J} 1_{[m] \backslash I \cup J}$, where $I, J$ are disjoint subsets of $[m]$. Set $D_{I} T_{J}:=D_{I} T_{J} 1_{[m] \backslash I \cup J}$. Now it can be easily seen that $\mathcal{Z}_{K}$ is cellular subcomplex of $\left(D^{2}\right)^{m}$ consisting of all cells $D_{I} T_{J}$ such that $I \in K$.

Lemma 3. The embedding $T^{m}=\rho^{-1}(1, \ldots, 1) \hookrightarrow \mathcal{Z}_{K}$ is a cellular map homotopic to the map to a point.

As it was shown in [2], for any field $\mathbf{k}$ there is the following isomorphism of algebras:

$$
H^{*}\left(\mathcal{Z}_{K}\right) \cong \operatorname{Tor}_{\mathbf{k}\left[v_{1}, \ldots, v_{m}\right]} \mathbf{k}(K), \mathbf{k}=H^{*} \mathbf{k}(K) \otimes \Lambda\left[u_{1}, \ldots, u_{m}\right], d,
$$

Partially supported by the Russian Foundation for Fundamental Research grant no. 99-01-00090. 
where $\mathbf{k}(K)$ is the Stanley-Raisner ring of complex $K$, and the differential $d$ is defined by $d\left(v_{i}\right)=0, d\left(u_{i}\right)=v_{i}, i=1, \ldots, m$. The Tor-algebra from (1) is naturally a bigraded algebra with $\operatorname{bideg}\left(v_{i}\right)=(0,2), \operatorname{bideg}\left(u_{i}\right)=(-1,2)$. The calculation of the $\operatorname{ring} H^{*}\left(\mathcal{Z}_{K}\right)$ allowed to describe the multiplicative structure in the cohomology of the complement of a coordinate subspace arrangement in $\mathbb{C}^{m}[2]$.

In [1] there was introduced the subcomplex $\mathcal{C}^{*}(K) \subset \mathbf{k}(K) \otimes \Lambda\left[u_{1}, \ldots, u_{m}\right]$ spanned by monomials $u_{J}$ and $v_{I} u_{J}$ such that $I \cap J=\varnothing, I \in K$. It was also shown there that the cohomology of $\mathcal{C}^{*}(K)$ is also isomorphic to that of $\mathcal{Z}_{K}$. Denote by $\mathcal{C}_{*}\left(\mathcal{Z}_{K}\right)$ and $\mathcal{C}^{*}\left(\mathcal{Z}_{K}\right)$ the chain and the cochain complex for type a) cellular decomposition of $\mathcal{Z}_{K}$ respectively.

Theorem 4. Let $\left(D_{I} T_{J}\right)^{*} \in \mathcal{C}^{*}\left(\mathcal{Z}_{K}\right)$ denote the cellular cochain dual to the cell $D_{I} T_{J} \in$ $\mathcal{Z}_{K}$. The correspondence $v_{I} u_{J} \mapsto\left(D_{I} T_{J}\right)^{*}$ defines a canonical isomorphism of complexes $\mathcal{C}^{*}(K)$ and $\mathcal{C}^{*}\left(\mathcal{Z}_{K}\right)$, each of which calculates $H^{*}\left(\mathcal{Z}_{K}\right)$.

The pair $\left(\mathcal{Z}_{K}, T^{m}\right)$ acquires a bigraded cellular structure by setting $\operatorname{bideg}\left(D_{i}\right)=(0,2)$, $\operatorname{bideg}\left(T_{i}\right)=(-1,2), \operatorname{bideg}\left(1_{i}\right)=(0,0)$. Put $b_{-q, 2 p}\left(\mathcal{Z}_{K}, T^{m}\right)=\operatorname{dim} H_{-q, 2 p}\left[\mathcal{C}_{*}\left(\mathcal{Z}_{K}, T^{m}\right)\right]$. Consider the new cellular decomposition of the poly-disc $\left(D^{2}\right)^{m}$ that is obtained by subdividing each factor $D^{2}$ into 5 cells $D, T, I, 1,0$, see Fig. b). This allows to introduce a bigraded cellular structure on $\mathcal{W}_{K}$ and define the numbers $b_{q, 2 p}\left(\mathcal{W}_{K}\right)=\operatorname{dim} H_{q, 2 p}\left[\mathcal{C}_{*}\left(\mathcal{W}_{K}\right)\right]$. Put

$$
\chi\left(\mathcal{Z}_{K}, T^{m} ; t\right)=\sum_{p, q}(-1)^{q} b_{-q, 2 p}\left(\mathcal{Z}_{K}, T^{m}\right) t^{2 p}, \quad \chi\left(\mathcal{W}_{K} ; t\right)=\sum_{p, q}(-1)^{q} b_{q, 2 p}\left(\mathcal{W}_{K}\right) t^{2 p}
$$

Let $f_{i}$ be the number of $i$-simplices of $K$, and $\left(h_{0}, \ldots, h_{n}\right)$ the $h$-vector of $K$ determined from the equation $h_{0} t^{n}+\ldots+h_{n-1} t+h_{n}=(t-1)^{n}+f_{0}(t-1)^{n-1}+\ldots+f_{n-1}$.

Theorem 5. Put $h(t)=h_{0}+h_{1} t+\cdots+h_{n} t^{n}$. Then

$$
\begin{aligned}
\chi\left(\mathcal{Z}_{K}, T^{m} ; t\right) & =\left(1-t^{2}\right)^{m-n} h\left(t^{2}\right)-\left(1-t^{2}\right)^{m}, \\
\chi\left(\mathcal{W}_{K} ; t\right) & =\left(1-t^{2}\right)^{m-n} h\left(t^{2}\right)+(-1)^{n-1} h_{n}\left(1-t^{2}\right)^{m} .
\end{aligned}
$$

Lemma 6. If $|K| \cong S^{n-1}$ (i.e., $K$ is a simplicial sphere), then $\mathcal{Z}_{K}$ is a closed manifold.

Suppose now that $K^{n-1}$ is a simplicial manifold. Then the complex $\mathcal{Z}_{K}$ generally fails to be a manifold. However, removing from $\mathcal{Z}_{K}$ a small neighbourhood $U_{\varepsilon}\left(T^{m}\right)$ of the orbit $\rho^{-1}(1, \ldots, 1) \cong T^{m}$ we obtain manifold $W_{K}=\mathcal{Z}_{K} \backslash U_{\varepsilon}\left(T^{m}\right)$ with boundary $\partial W_{K}=|K| \times T^{m}$.

Theorem 7. The manifold (with boundary) $W_{K}$ is equivariantly homotopy equivalent to the complex $\mathcal{W}_{K}$. There is a canonical homeomorphism of pairs $\left(W_{K}, \partial W_{K}\right) \rightarrow\left(\mathcal{Z}_{K}, T^{m}\right)$.

The relative Poincaré duality isomorphisms for $W_{K}$ imply

$$
\chi\left(\mathcal{W}_{K} ; t\right)=(-1)^{m-n} t^{2 m} \chi\left(\mathcal{Z}_{K}, T^{m} ; \frac{1}{t}\right) .
$$

Taking into account Theorem 5 we obtain

Corollary 8. Let $K^{n-1}$ be a simplicial manifold. Then

$$
h_{n-i}-h_{i}=(-1)^{i}\left(h_{n}-1\right){ }_{i}^{n}=(-1)^{i} \chi\left(K^{n-1}\right)-\chi\left(S^{n-1}\right) \quad \begin{gathered}
n \\
i
\end{gathered}, \quad i=0,1, \ldots, n,
$$

where $\chi(\cdot)$ denotes the Euler number.

Rewriting the equation (8) in terms of the $f$-vector we come to more complicated equations, which were deduced in [4], [5]. For $|K|=S^{n-1}$ Corollary 8 gives the classical Dehn-Sommerville equations. In the particular case of PL-manifolds the topological invariance of numbers $h_{n-i}-h_{i}$ (which follows directly from Corollary 8) was firstly observed by Pachner in $[6,(7.11)]$.

The extended version of this article is http://xxx.lanl.gov/abs/math.AT/0005199. The authors wish to express special thanks to Oleg Musin for stimulating discussions and helpful comments, in particular, for drawing our attention to the results of [4] and [6]. 


\section{REFERENCES}

[1] V.M. Buchstaber and T.E. Panov. Proceedings of the Steklov Institute of Mathematics 225 (1999), 87-120.

[2] V. M. Buchstaber and T. E. Panov. (Russian), Zap. Nauchn. Semin. POMI 266 (2000), 29-50.

3] M. A. Shtan'ko and M. I. Shtogrin. Russian Math. Surveys 47 (1992), no. 1, 267-268.

[4] B. Chen, M. Yan. Proceedings of the Steklov Institute of Mathematics 221 (1998), 305-319.

[5] V. Klee. Canadian J. Math. 16 (1964), 517-531.

[6] U. Pachner. European J. Combinatorics 12 (1991), 129-145.

Department of Mathematics and Mechanics, Moscow State University, 119899 Moscow, RUSSia

E-mail address: buchstab@mech.math.msu.su tpanov@mech.math.msu.su 